EPJ Web of Conferences 47, 12001 (2013)

DOI: $10.1051 /$ epjconf/20134712001

(C) Owned by the authors, published by EDP Sciences, 2013

\title{
Mechanisms affecting the composition of Hot Jupiters atmospheres
}

\author{
Vivien Parmentier ${ }^{1, \mathrm{a}}$, Tristan Guillot ${ }^{1, \mathrm{~b}}$ and Adam P. Showman ${ }^{2, \mathrm{c}}$ \\ ${ }^{1}$ Université de Nice-Sophia Antipolis, Observatoire de la Côte d'Azur, CNRS UMR 6202, \\ BP. 4229, 06304 Nice Cedex 4, France \\ ${ }^{2}$ Department of Planetary Sciences, Lunar and Planetary Laboratory, University of Arizona, \\ Tucson AZ, USA
}

\begin{abstract}
Opacities and thus local chemical composition play a key role when characterizing exoplanet atmospheres from observations. When the gas is in chemical equilibrium the chemical abundances depend strongly on the temperature profile. Grey models tend to overestimate the temperatures in the upper atmosphere. We present a new analytical model with a more realistic description of the radiative cooling in the infrared. Mechanisms like quenching and cold traps can drive the upper atmosphere far from its chemical equilibrium. The efficiency of these mechanisms depends on the strength of the vertical mixing. Using 3D global circulation models of HD209458b including passive tracers, we show that, although Hot Jupiter atmospheres are stably stratified, they are strongly mixed by planetary scale circulation patterns. We provide a rough estimate of the effective vertical mixing coefficient in Hot Jupiter atmosphere which can be used in 1D models.
\end{abstract}

\section{INFRARED COOLING OF THE UPPER ATMOSPHERE}

1D analytical radiative transfer models of planetary atmospheres are useful when addressing problems where a wide range of parameter have to be tested. Up to now, analytical models for irradiated planets were derived by assuming different opacities in the visible and in the infrared (this is the semi-grey assumption) but a constant opacity in the infrared [1-3]. However, as can be seen in Fig. 1, the semigrey models systematically overestimate the temperature in the upper atmosphere.

Following the work of Chandrasekhar [5], we derive a new 1D radiative model of irradiated atmosphere taking into account the non-grey effects in the infrared. We solve the radiative transfer equations following [2] using a comb-like opacity in the infrared instead of the grey approximation. We validate our solution by comparing it with a the 1D numerical code originally developed by [6]. Our model allows a better fit of the numerical solutions using the full line by line opacities as can be seen by the green line in Fig. 1. We correctly reproduce the cooling of the upper atmosphere. The strength of the cooling is determined principally by the ratio of the Planck mean to the Rosseland mean opacity, $\gamma_{\mathrm{p}}=\kappa_{\mathrm{p}} / \kappa_{\mathrm{r}}$. This ratio is equal to 1 for a grey atmosphere and is always greater than 1 for non-grey opacities, making it a good measurement of the grayness of the atmosphere [7]. The skin temperature i.e. the temperature at the top of the atmosphere is a decreasing function of $\gamma_{p}$. For arbitrarily high values of $\gamma_{p}$, the upper atmosphere can cool to arbitrarily low temperatures as can be seen in Fig. 2. Our model provide a physically motivated temperature profile that explore a wider range of parameters than the

\footnotetext{
ae-mail: vivien. parmentier@oca.eu

be-mail: tristan.guillot@oca.eu

${ }^{c}$ e-mail: showman@lpl.arizona.edu
}

This is an Open Access article distributed under the terms of the Creative Commons Attribution License 2.0, which permits unrestricted use, distribution, and reproduction in any medium, provided the original work is properly cited. 


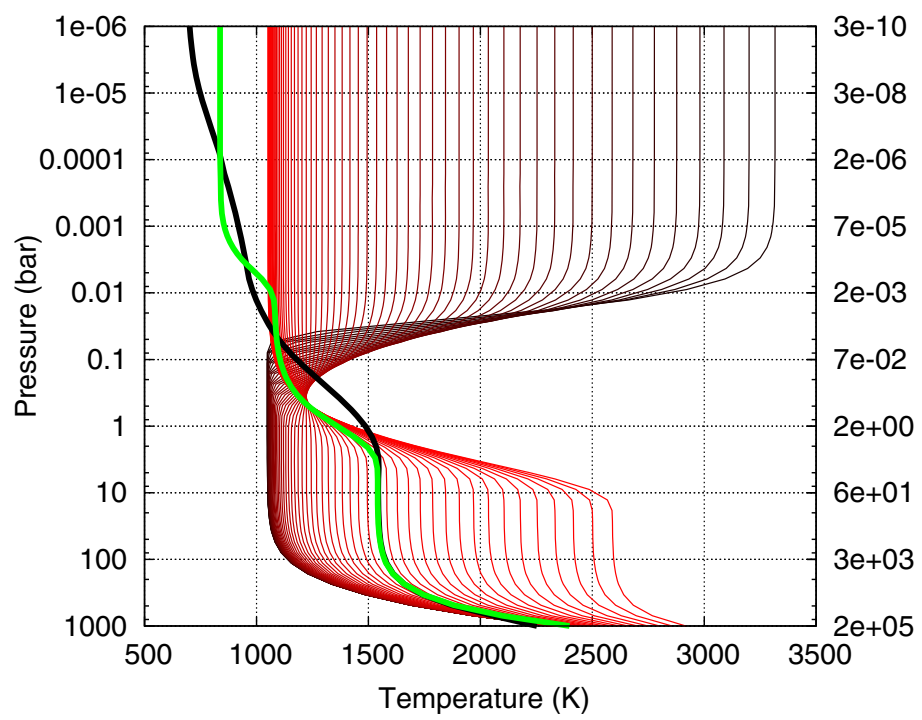

Figure 1. Semi-grey solution for visible opacities ranging from 0.01 to 100 times the infrared opacity (thin lines). Models for small visible opacities are redder. The black thick line is a numerical profile calculated using the line by line opacities of [4]. The green line is our non-grey analytical model using $\gamma_{\mathrm{p}}=8$. We used $T_{e q}=1244 \mathrm{~K}, \mu=0.5$ and dividing the incident flux by two for a dayside average. TiO and $\mathrm{VO}$ opacities are not considered.

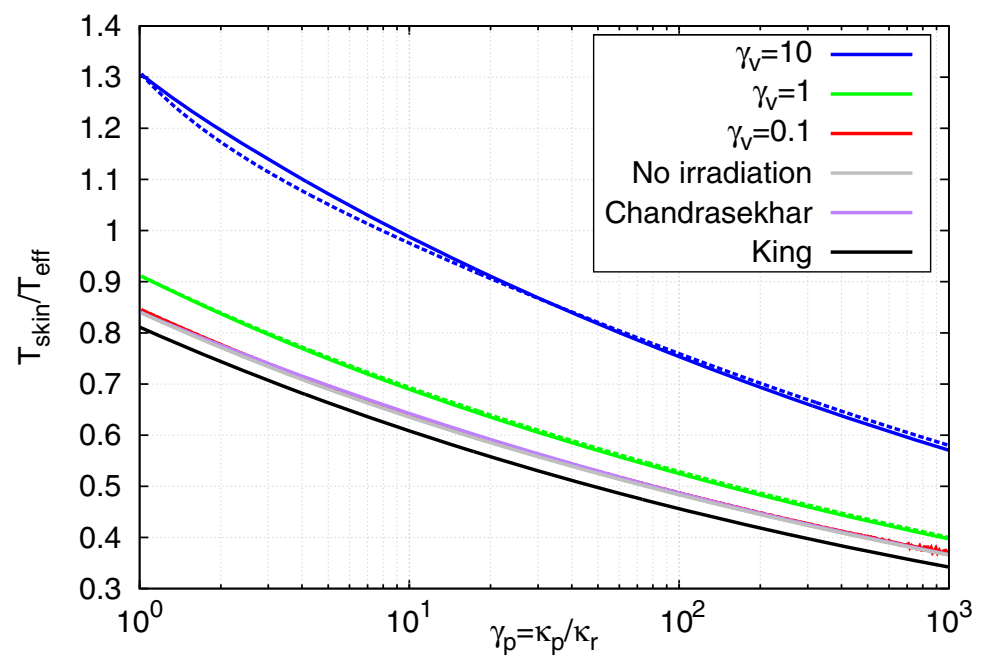

Figure 2. Skin temperature of the planet given by our model for different ratio of the visible to the thermal opacity $\gamma_{v}$ in function of $\gamma_{p}$ for lines covering $1 \%$ (plain lines) or 10\% (dashed lines) of the wavelength range. Also plotted are the models of Chandrasekhar [5] and King [7] that were derived without irradiation.

semi-grey model. The model is presented in more details in [8] and is available for download at the address: http://www.oca.eu/parmentier.

\section{3D MIXING IN HOT JUPITER ATMOSPHERES}

Hot Jupiter circulation is driven by the strong day/night contrast in the irradiation they receive from their parent star. Despite the fast winds that carry heat from day to night, they are believed to harbor a 

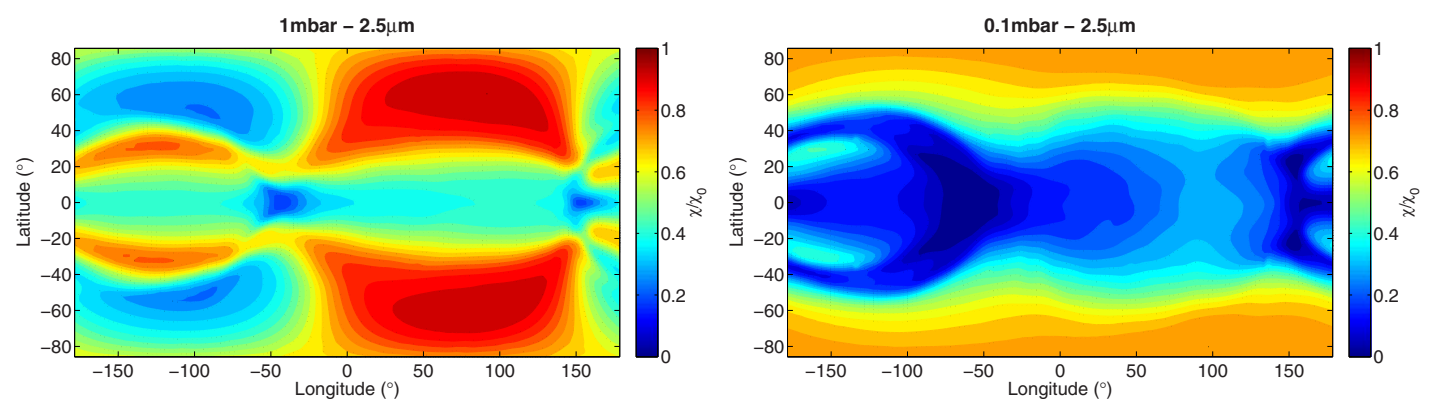

Figure 3. Time averaged tracer abundance at two different pressure for a condensate size of $2.5 \mu \mathrm{m}$.

day/night temperature contrast that can reach $\sim 1000 \mathrm{~K}$ [9]. Several gaseous species, including titanium and vanadium oxides and silicates, can be stable on the warm dayside but should condense on the cold night side of the planet. The particles on the night side could therefore settle down and deplete the whole planet in a given chemical species. The actual abundances of a chemical species at a given pressure level depends on the competition between settling on the night side and vertical mixing due to the atmospheric circulation.

We implement tracers in a 3D global circulation model (GCM, see [9]) of HD209458b to model a chemical species that condenses and settles on the nightside of the planet. This is the first study with a representation of clouds in a global circulation model of hot Jupiters [10].

We run the model for 1400 days and average the last 400 days of simulation, once the model reaches a statistical steady-state. The spatial distribution of the tracers is inhomogeneous. Around 0.1 mbar, the tracer field is rather independent of longitude and the equatorial band is more depleted than the poles whereas at 1 mbar, a day/night contrast on the tracer abundance appears at high latitudes (see Fig. 3).

The model also exhibits up to $75 \%$ temporal variability in the tracer abundances with timescales ranging from days to hundreds of days (see Fig. 4). This variability depends on the assumed size of the condensate particles. The bigger the particles the stronger the variability.

This spatiotemporal variability in the tracer field can have interesting consequences during transit and secondary eclipse observations. Our model can be applied to radiatively active molecules such as titanium oxide $(\mathrm{TiO})$, the most cited candidate for producing the inferred temperature inversions in hot Jupiters. Temporal variability of $\mathrm{TiO}$ abundances in the upper atmosphere of hot Jupiters could lead some planets to oscillate between states with and without stratospheres, what will strongly affect the dayside emission of the planet. Our model also suggest that some planets might have a spatial and temporal variability in their cloud coverage, leading to a longitudinal and latitudinal variations in their albedo.

Most 1D models of hot Jupiter atmospheres dealing with disequilibrium processes or vertical transport consider the vertical mixing as a diffusive process and need to assume a value for $\kappa_{\mathrm{zz}}$, the $1 \mathrm{D}$ vertical diffusion coefficient. However, no there is no theoretical reason to believe that the transport induced by the 3D circulation in hot Jupiters can be approximated by a $1 \mathrm{D}$ vertical diffusion process. It is therefore difficult to estimate a priori the vertical diffusion coefficient. Though, from the 3D model we estimate $a$ posteriori the effective vertical diffusion coefficient of the simulation:

$$
K_{\mathrm{zz}}=\frac{5 \times 10^{4}}{\sqrt{P_{\mathrm{bar}}}} \mathrm{m}^{2} \mathrm{~s}^{-1} .
$$

This value can be used to guide 1D modelers in their choice of parameters. It represents the vertical mixing induced by the large scale circulation of the planet, resolved by the GCM, and not the small scale molecular and turbulent mixing that is not taken into account in our model. However, giving the 


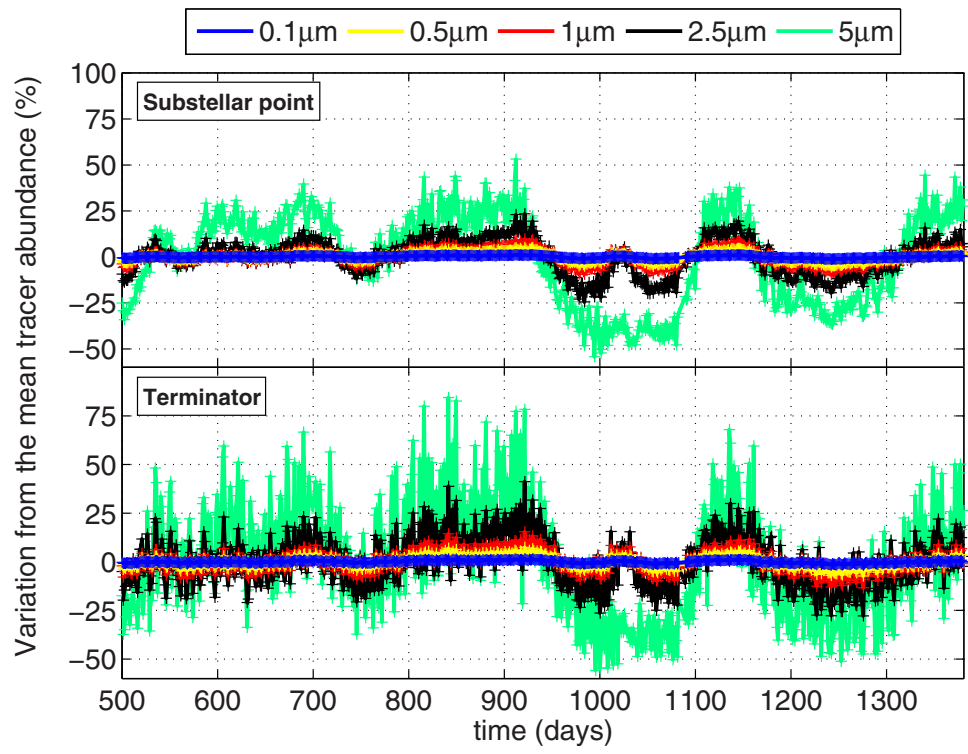

Figure 4. Time dependence of the mean tracer abundance between 1 mbar and 0.1 mbar, within $45^{\circ}$ around the substellar point (top) and between $+5^{\circ}$ and $-5^{\circ}$ around the terminator (bottom) for different sizes of the nightside condensate. The top panel is relevant for inferring the variation in the strength of the stratosphere, albedo variations, or secondary eclipse measurements. The bottom panel is relevant for transit spectroscopy measurements.

strong mixing induced by the circulation, we believe that the large scale circulation is the dominant mixing mechanism in hot Jupiters.

\section{References}

[1] B.M.S. Hansen, ApJS 179, 484 (2008), 0801.2972

[2] T. Guillot, A\&A 520, A27+ (2010), 1006.4702

[3] T.D. Robinson, D.C. Catling, ApJ 757, 104 (2012), 1209.1833

[4] R.S. Freedman, M.S. Marley, K. Lodders, ApJS 174, 504 (2008), 0706.2374

[5] S. Chandrasekhar, MNRAS 96, 21 (1935)

[6] C.P. McKay, J.B. Pollack, R. Courtin, Icarus 80, 23 (1989)

[7] I.J.I.F. King, ApJ 121, 711 (1955)

[8] V. Parmentier, T. Guillot, J.J. Fortney (2013), in prep.

[9] A.P. Showman, J.J. Fortney, Y. Lian, M.S. Marley, R.S. Freedman, H.A. Knutson, D. Charbonneau, ApJ 699, 564 (2009), 0809. 2089

[10] V. Parmentier, A.P. Sowman, J.J. Fortney (2013), in prep. 